\title{
Descripción de cuatro especies nuevas del subgrupo Drosophila fasciola, grupo repleta (Diptera, Drosophilidae) en dos bosques nublados del Ecuador
}

\author{
María Beatriz Cabezas, Luz Marina Llangarí \& Violeta Rafael
}

Laboratorio de Genética Evolutiva, Escuela de Ciencias Biológicas, Pontificia Universidad Católica del Ecuador, 17-01-2184, Quito, Ecuador (mabec1306@hotmail.com; focoacuatico@hotmail.com; vrafael@puce.edu.ec)

\begin{abstract}
Description of four new species of the Drosophila fasciola subgroup, repleta group (Diptera, Drosophilidae) in two cloud forests of Ecuador. The current study describes four new members of the Drosophila repleta group, collected in two cloud forests. The new species were grouped into the $D$. fasciola subgroup, based on thorax pigmentation patterns and male genitalia morphology. Drosophila inti sp. nov., D. nigua sp. nov. and D. yambe sp. nov. were collected using banana baits, while D. carvalhoi sp. nov. was collected from Anthurium sp. inflorescences. The abdominal pigmentation and shape of the edeago of $D$. nigua sp. nov., D. yambe sp. nov. y D. carvalhoi sp. nov. suggest a close relationship between them, whereas $D$. inti sp. nov. would be more closely related to $D$. linearepleta Patterson \& Wheeler, 1942.
\end{abstract}

KEYWORDS. Intillacta, Guajalito, taxonomy, genitalia, Anthurium.

RESUMEN. En el presente trabajo se describen cuatro miembros nuevos del grupo Drosophila repleta capturados en dos bosques nublados del Ecuador. Las nuevas especies fueron agrupadas dentro del subgrupo $D$. fasciola basados en el patrón de pigmentación del tórax y la morfología de la genitalia masculina. Drosophila inti sp. nov., D. nigua sp. nov. y D. yambe sp. nov. fueron capturadas con trampas de banano, mientras que $D$. carvalhoi sp. nov. fue recolectada en inflorescencias de Anthurium sp. La pigmentación del abdomen y la forma del edeago de $D$. nigua sp. nov., $D$. yambe sp. nov. y $D$. carvalhoi $\mathrm{sp}$. nov. indicarían que están relacionadas entre ellas, mientras que $D$. inti sp. nov. sería más cercana a $D$. linearepleta Patterson \& Wheeler, 1942.

PALABRAS-CLAVE. Intillacta, Guajalito, taxonomía, genitalia, Anthurium.

Los bosques nublados son denominados así debido a la humedad que se eleva desde las zonas bajas, provocando una densa neblina y alcanzando su mayor diversidad biológica entre los 1000 - 3500 msnm (DANGLes et al., 2009). La investigación sobre la biodiversidad en los bosques nublados con cualquier grupo biológico es de vital importancia, más aún si se trata de organismos que ocupan una posición central, que permiten estudiar la diversidad de las comunidades y la conservación de los ecosistemas como es el caso del género Drosophila (CAVASINI et al., 2008).

Uno de los grupos del género Drosophila, es el grupo D. repleta Sturtevant, 1942, endémico de América (Silva-Bernardi et al., 2006), y además uno de los más numerosos y complejos del neotrópico (Suyo \& Pilares, 1988; Silva-Berdani et al., 2006). Actualmente se conoce al menos 102 especies descritas a lo largo del continente Americano (GotTsChaLK et al., 2006). Los miembros de este grupo se caracterizan principalmente por tener un mesonoto gris o marrón, con pelos y cerdas emergiendo de un punto negro o marrón oscuro, alas con venas longitudinales claras y generalmente no presentan dientes secundarios (VILELA, 1983).

La identificación del género Drosophila se ha realizado principalmente a través del estudio de la genitalia masculina, cuya arquitectura es la más vulnerable genéticamente, y en el proceso de especiación, es la primera estructura que experimenta una separación intraespecífica (Suyo et al., 1988). En el grupo D. repleta existen seis subgrupos ellos son: D. repleta Sturtevant, 1942, D. hydei Wharton, 1944, D. mercatorum Wharton, 1944, D. mulleri Wharton, 1944, D. fasciola Wasserman, 1962 y D. inca Rafael \& Arcos, 1989.

THROCKMORTON (1982) discutió el origen del grupo $D$. repleta, y comentó que es difícil determinar si el ancestro del grupo tuvo su origen dentro de un bosque, y luego comenzó a diversificarse y colonizó los hábitats secos, o viceversa.

Las especies del grupo D. repleta, ocupan zonas áridas y semiáridas, muchas de ellas utilizan cactáceas, frutas en descomposición como sustrato trófico y también pueden estar en ambientes domésticos (SuYo et al., 1988). El subgrupo D. fasciola fue el primero en aparecer en la literatura en un artículo previo escrito por Wasserman, 1960 (VILELA, 1983). Este subgrupo es el único, dentro del grupo $D$. repleta, con la mayoría de sus miembros viviendo en bosques húmedos de Centro y Sudamérica, y comprende 21 especies (Silva-BERnARDi et al., 2006). Los miembros de este subgrupo se caracterizan principalmente por presentar manchas en el mesonoto, margen anterior del cerci fusionados al borde posterior del epandrio en diversos grados, surestilo sin dientes secundarios, y el edeago por lo general con un par de espuelas en la parte ventral posterior (VILELA, 1983).

ACURIO \& RAFAEL (2009) mencionaron 28 especies del grupo D. repleta: $D$. aldrichi Patterson and Crow, 1940; D. ellisoni Vilela, 1983; D. fasciola Williston, 1896; D. 
huayla Suyo, Pilares \& Vásquez, 1988; D. hydei Sturtevant, 1921; D. inca Dobzhansky \& Pavan, 1943; D. longicornis Patterson \& Wheeler, 1942; D. mayaguana Vilela, 1983; D. mercatorum Patterson \& Wheeler, 1942; D. meridiana Patterson \& Wheeler, 1942; D. meridionalis Wasserman, 1962; D. mojuoides Wasserman, 1962; D. neohydei Wasserman, 1962; D. neorepleta Patterson \& Wheeler, 1942; D. nigricruria Patterson \& Wheeler, 1942; D. nigrohydei Patterson \& Wheeler, 1942; D. nigrospiracula Patterson \& Wheeler, 1942; D. paranaensis Barros, 1950; D. parisiena Heed \& Grimaldi, 1991; D. peninsularis Patterson \& Wheeler, 1942; D. pseudorepleta Vilela \& Bächli, 1990; D. repleta Sturtevant, 1921; D. straubae Heed \& Grimaldi, 1991; D. vicentinae Vilela, 1983; D. guayllabambae Rafael \& Arcos, 1988; D. yangana Rafael \& Vela; 2003; D. shuyu Vela \& Rafael, 2005; D. huancavilcae Rafael \& Arcos, 1988. Las cuatro últimas especies han sido descubiertas en Ecuador.

El propósito del presente trabajo fue ampliar el conocimiento de las especies del grupo $D$. repleta en dos bosques nublados ecuatorianos.

\section{MATERIALES Y MÉTODOS}

Se realizaron muestreos en dos bosques nublados durante el 2011. El bosque nublado Reserva Intillacta $\left(00^{\circ} 03^{\prime} 01.6\right.$ 'N, 078 43'23.7' W, 1700-1890 msnm) está ubicado en la zona noroccidental de la Provincia de Pichincha dentro del Bosque Protector Mindo-Nambillo, kilómetro 63,5 de la vía Calacalí - La Independencia, en el camino secundario hacia el recinto Miraflores. Y el Bosque nublado Estación Científica Río Guajalito $\left(00^{\circ} 13^{\prime} 48.8^{\prime}\right.$ 'S 078 49' 16.5' 'W, 1800-2200 msnm), ubicado en la zona centro occidental de la Provincia de Santo Domingo de los Tsáchilas, cerca de la localidad Las Palmeras, entre Chiriboga y el Empalme.

Para las capturas se utilizaron botellas plásticas conteniendo cebo preparado según describe RAFAEL et al., (2000), y permanecieron de 8 a 10 días en el campo.

En el Bosque nublado Estación Científica Río Guajalito también se realizó capturas de moscas Drosophila en inflorescencias de Anthurium y Xanthosoma (Araceae). La recolección de drosófilas se llevó a cabo en las primeras horas de la mañana y de la tarde. Para ello se utilizó un aspirador entomológico.

Las moscas capturadas se colocaron en tubos de vidrio con medio de cultivo. Cada hembra fue colocada en un tubo con medio de cultivo, con la finalidad de obtener descendientes que constituirían las isolíneas. Estas isolíneas permitieron identificar ambos sexos de cada especie.

Cuando moría un individuo, el cuerpo fue colocado en alcohol para el posterior análisis. Para examinar la genitalia se extrajo los dos últimos segmentos abdominales, y fueron colocados individualmente en un microvial conteniendo una solución de hidróxido de potasio al $10 \%$ y luego sometidos a cocción por 10 a 15 minutos, e inmediatamente se separó las estructuras genitálicas, arco genital, hipandrio y edeago en los machos; oviscapto y espermatecas en las hembras. Estas estructuras fueron observadas en un microscopio.

Una vez terminado el análisis, las especies nuevas fueron descritas (holotipo, alotipo y paratipos). Estos individuos se dejaron al ambiente para que se sequen y luego fueron montados en triángulos entomológicos con sus correspondientes estructuras genitálicas. Los especímenes analizados fueron depositados en el Museo de Zoología, sección Invertebrados de la Pontificia Universidad Católica del Ecuador, Quito (QCAZ-I). La nomenclatura de las estructuras descritas fueron tomadas de B̈̈CHLI et al. (2004), y los índices de las espermatecas son los propuestos por RAFAEL \& ARCOS (1989).

\section{RESULTADOS}

\section{Drosophila inti sp. nov.}

(Figs 1-5)

Material tipo. Holotipo $\widehat{\partial}$ (disectado, genitalia en microtubo, montado en seco), ECUADOR, Pichincha: (Intillacta, 0003'01.6” N, 78 43'23.7' W, $1890 \mathrm{msnm}$ ), II.2011, M. B. Cabezas col., V. Rafael \& M. B. Cabezas

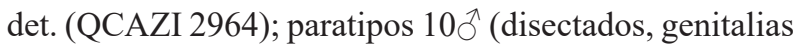
en microtubo, montados en seco) los mismos datos del holotipo (QCAZI 2965-2974).

Diagnosis. Color del cuerpo amarrillento. Tórax con cerdas y pelos emergiendo de puntos oscuros. Abdomen amarillo, primer tergito ligeramente pigmentado, presenta línea media dorsal del segundo al sexto tergito. Edeago formado por dos láminas quitinizadas, con una abertura dorsal que alcanza casi toda la longitud del edeago y luego se continúa con una sutura, en la parte dorsal del extremo distal presenta estrías.

Descripción del macho, holotipo (individuo en alcohol). Morfología externa: longitud total (cuerpo + alas) $4.00 \mathrm{~mm}$, longitud del cuerpo $2.60 \mathrm{~mm}$. Color del cuerpo amarillento.

Cabeza. Arista plumosa, segundo y tercer segmento de las antenas marrón amarillento, con cuatro ramas dorsales y dos ventrales, más la terminal bifurcada. Cabeza con placa orbital marrón claro, longitud frontal $0.50 \mathrm{~mm}$; índice frontal 1.66; radio de disminución frontal 1.56; cerda orbital media más cerca a la cerda orbital anterior y del borde de la placa orbital, índice vt 1.11; distancia or1/or3 0.17 ; distancia or2/or1 0.07 . Triángulo ocelar marrón oscuro, ocelos amarillos; triángulo frontal marrón claro, frontal vitta marrón claro, gena y postgena marrón amarillento, índice genal 3.00; índice vibrisa 0.50. Carina prominente surcada, marrón amarillento. Proboscis amarillenta. Ojos de color rojo vinoso, índice ocular 1.45.

Tórax. Amarillo, con cerdas y pelos emergiendo de puntos oscuros, con ocho hileras de pelos acrosticales entre las cerdas dorsocentrales anteriores, índice h 0.89 ; índice dc 1.75. En la parte anterior del tórax se observa dos franjas 

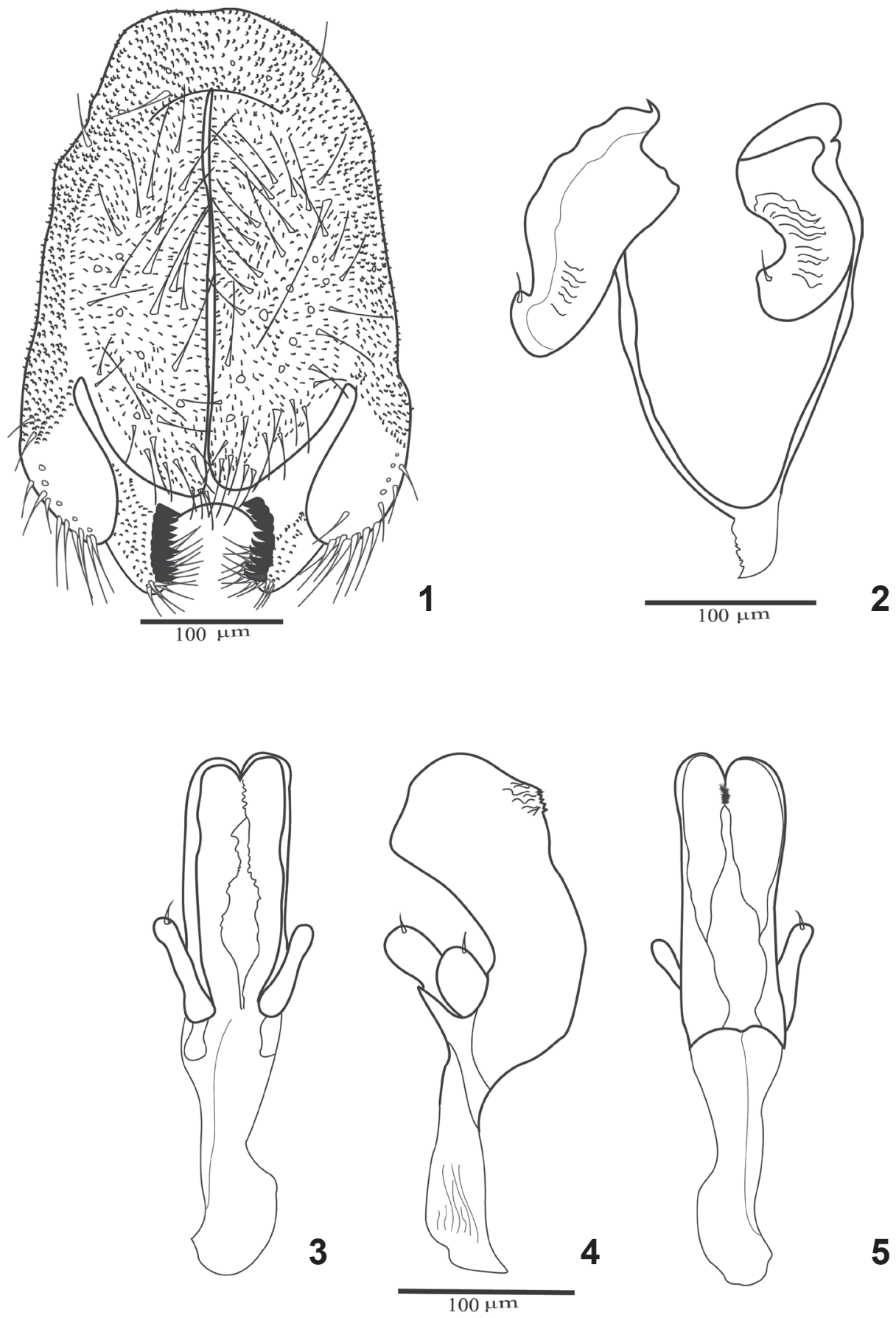

5

Figs 1-5. Drosophila inti sp. nov., holotipo ổ: 1, arco genital; 2, hipandrio; edeago: 3, vista ventral; 4, vista lateral; 5, vista dorsal.

oscuras que alcanzan la parte media, existe una banda oscura en la parte externa de las dorsocentrales anteriores, lóbulo prescutal amarillo rodeado de un área oscura, sutura prescutal con una banda oscura en la parte dorsal. Escutelo marrón con el borde amarillo; cerdas escutelares anteriores convergentes emergiendo de puntos oscuros, índice scut 0.77; índice sterno 0.60. La zona esternopleural presenta dos bandas oscuras a lo largo de las esternopleuras. Patas, coxa y fémur marrón amarillento, primer par de patas con un anillo marrón apenas perceptible en la parte inicial del fémur el resto amarillo, en el segundo y tercer par de patas el anillo es evidente; celda costal y celda marginal ligeramente más pigmentada, vena transversal posterior poco sombreada, lóbulo costal ligeramente pigmentado. Longitud del ala $2.88 \mathrm{~mm}$. Índices alares: alar 1.95; C 2.55 ; ac 2.15; hb 0.50; 4c 0.86; 4v 1.52; 5x 1.15; M 0.48 y prox. x 0.68 .

Abdomen. Color amarillo, primer tergito ligeramente pigmentado, presenta línea media dorsal del segundo al sexto tergito, segundo tergito con pigmentación en la parte 
posterior que se adelgaza hacia los lados, en la parte lateral presenta un área clara, que luego se pigmenta a todo lo ancho; tercer tergito igual al anterior con un área clara apenas perceptible en la parte lateral, cuarto y quinto tergito con pigmentación en la parte posterior se adelgaza hacia los lados y nuevamente se pigmenta a todo lo ancho del tergito; sexto tergito ligeramente pigmentado.

Terminalia. Cerci unidos al epandrio. Epandrio microtricoso, con siete cerdas en la parte dorsal, una cerda en la parte media del lado izquierdo mientras que en el lado derecho no presenta, en la parte baja del epandrio con 16 cerdas en ambos lados. Decasterno rectangular sin hendidura. Surestilo microtricoso y 10 dientes primarios en ambos lados y 14 cerdas marginales (Fig. 1). Hipandrio de forma triangular y membranoso (roto). Gonopodio poco quitinizado alargado y estriado, con una cerda en la parte interna (Fig. 2). Edeago quitinizado, formado por dos láminas. En vista dorsal se observa la abertura dorsal que ocupa casi toda la longitud del edeago y luego se continúa con una sutura, en la parte dorsal del extremo distal presenta estrías (Figs 3-5). Rama ventral quitinizada desarrollado. Paráfisis alargada con una cerda en el extremo distal. Pie del edeago poco quitinizado, membranoso. Índice del edeago 1.14.

Variación en paratipos (individuos en alcohol). Morfología externa: longitud total (cuerpo + alas) 3.95$4.39 \mathrm{~mm}$. Longitud frontal $0.50-0.51 \mathrm{~mm}$; índice frontal 1.66-1.67; radio de disminución frontal 1.54-1.56); índice vt 1.05-1.11; distancia or1/or3 $0.15-0.17$; distancia or $2 /$ or1 0.05-0.07; índice genal 3.00-3.05; índice vibrisa 0.490.52; índice ocular 1.45-1.47. Índice h 0.89-0.92; índice dc 1.75-1.76; índice scut 0.76-0.79; índice sterno 0.60-0.63. Epandrio con seis-siete cerdas en la parte dorsal, algunos individuos no presentan cerdas en la parte media y 1416 cerdas en la parte baja. Surestilo con 10-11 dientes primarios y 14-16 cerdas marginales. Índice del edeago 1.13-1.17.

Hembra: Desconocida.

Etimología. El nombre de la especie es en honor al dueño de la Reserva Intillacta, Sr. Inti Arcos. "Inti” es una palabra del idioma quichua que significa sol.

Relación con otras especies. Drosophila inti sp. nov. se encuentra estrechamente relacionada con $D$. linearepleta Patterson \& Wheeler, 1942 debido al gran parecido a nivel de la genitalia.

\section{Drosophila nigua sp. nov.}

$$
\text { (Figs 6-10) }
$$

Material tipo. Holotipo $\widehat{\partial}$ (disectado, genitalia en microtubo, montado en seco), ECUADOR, Pichincha: (Intillacta, 0003'01.6”'N, 078 43'23.7' W, $1890 \mathrm{msnm}$ ), II.2011, M. B. Cabezas col., V. Rafael \& M. B. Cabezas det. (QCAZI 2949); paratipos $6 \hat{0}$ (disectados, genitalias en microtubo, montados en seco) los mismos datos del holotipo, (QCAZI 2950-2955). Paratipo §̂ ECUADOR,
Santo Domingo de los Tsáchilas: (Guajalito, $00^{\circ} 13$ '48.8”S 07849'16.5”'W, 1890 msnm) VII.2011, M. B. Cabezas col., V. Rafael \& M. B. Cabezas det. (QCAZI 2953).

Diagnosis. Color del cuerpo amarrillento. Tórax con cerdas y pelos emergiendo de puntos marrones. Abdomen amarillo, con línea media dorsal. Primer tergito con una franja oscura en la parte posterior; los otros tergitos presentan pigmentaciones en la parte posterior y se adelgazan hacia los lados, luego las pigmentaciones alcanzan el borde anterior de los tergitos seguido por un espacio amarillo y nuevamente los tergitos se pigmentan a manera de triángulo. Parte distal del edeago triangular, con la punta ligeramente hendida, en la parte ventral con dos proyecciones laterales pequeñas como ganchos $(\mathrm{g}), \mathrm{y}$ dos proyecciones largas y delgadas (r.sa).

Descripción del macho, holotipo (individuo en alcohol). Morfología externa: longitud total (cuerpo + alas) $4.20 \mathrm{~mm}$, longitud del cuerpo $2.70 \mathrm{~mm}$. Color del cuerpo amarillento.

Cabeza. Marrón con áreas amarillas. Segundo y tercer segmento de las antenas marrón amarillento. Arista plumosa con cuatro ramas dorsales y dos ventrales, más la terminal bifurcada. Cabeza con placa orbital marrón, longitud frontal $0.40 \mathrm{~mm}$; índice frontal 1.21; radio de disminución frontal 1.63; la cerda orbital media más cerca a la cerda orbital anterior y del borde de la placa orbital, y nacen de puntos oscuros, índice vt 0.93 ; distancia orl/ or3 0.11 ; distancia or2/or1 0.07 . Triángulo ocelar marrón oscuro, ocelos amarillos; triángulo frontal de color marrón, frontal vitta marrón, gena y postgena marrón, índice genal 3.71; índice vibrisa 0.64. Carina prominente surcada, marrón amarillento. Proboscis y palpo amarillentas. Ojos de color rojo vinoso, índice ocular 1.02.

Tórax. Amarillo, con cerdas y pelos emergiendo de puntos marrones, con seis hileras de pelos acrosticales entre las cerdas dorsocentrales anteriores, índice h 1.00; índice de 0.75 , a lo largo de todo el tórax en dirección de las dorsocentrales hay una banda marrón oscura, en ambos lados de la banda se observa zonas claras, en la parte anterior del tórax existen dos bandas oscuras pequeñas. Lóbulo prescutal amarillo bordeado por una zona oscura. Escutelo color marrón amarillento con todo el borde amarillo; cerdas escutelares anteriores convergentes y emergiendo de puntos oscuros, índice scut 1.40; índice sterno 0.76. Patas coxa y fémur marrón oscuro, tibia presenta dos anillos uno en la parte inicial y otro en la parte final. Alas amarillas, vena transversal posterior poco sombreada, lóbulo costal oscuro. Longitud del ala $2.68 \mathrm{~mm}$. Índices alares: alar 1.97; C 2.30; ac 2.58; hb 0.52; 4c 1.00; 4v 1.78; 5x 1.19; M 0.52 y prox. x 0.66 .

Abdomen. Color amarillo, con línea media dorsal. Primer tergito con una franja oscura en la parte posterior, los otros tergitos también presentan pigmentaciones en la parte posterior y se adelgazan hacia los lados. Luego las pigmentaciones alcanzan el borde anterior de los tergitos y son seguidos por un espacio amarillo y nuevamente cada tergito se pigmenta a manera de triángulo. 

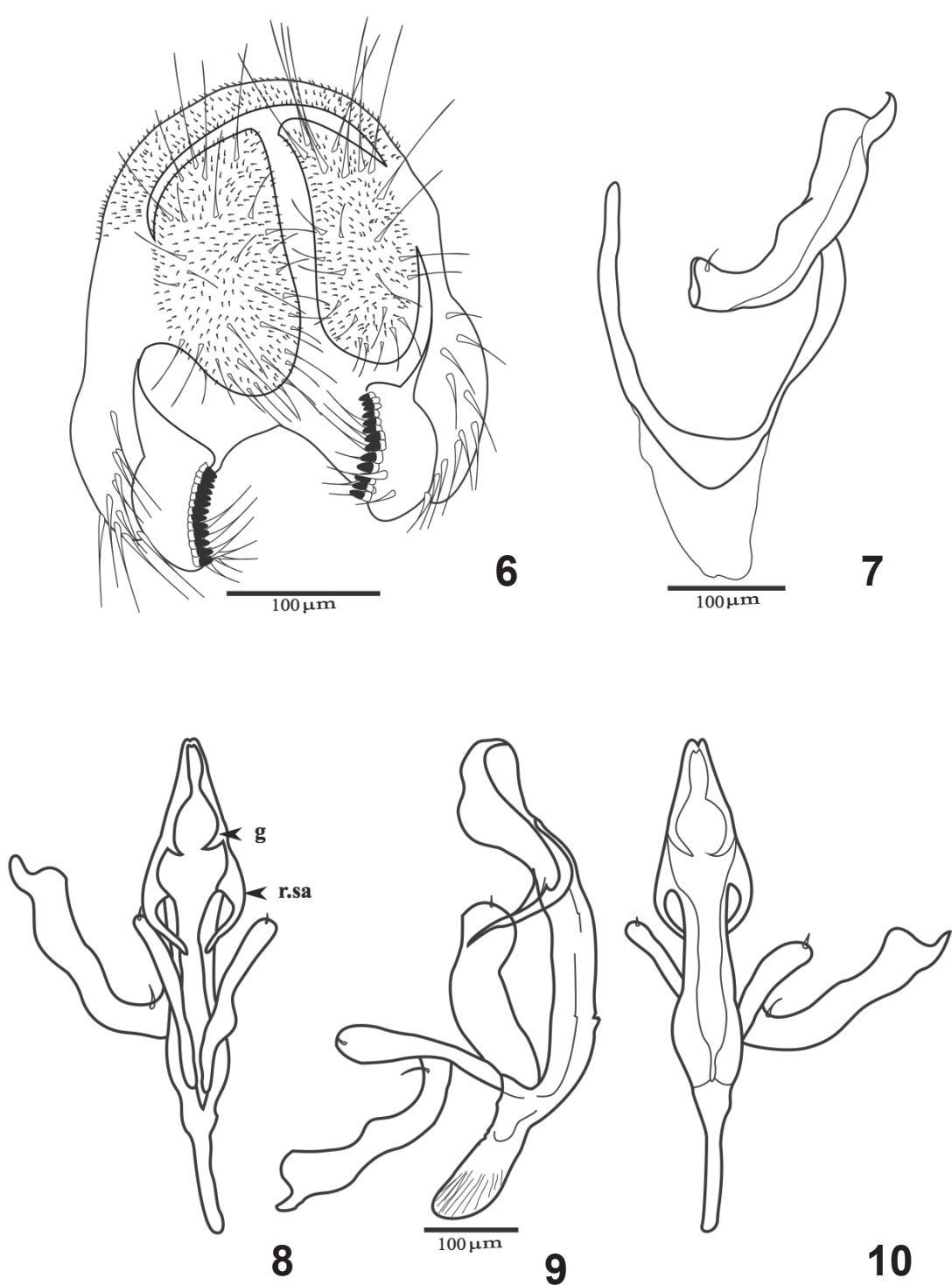

10

Figs 6-10. Drosophila nigua sp. nov., holotipo 3 : 6, arco genital; 7, hipandrio; edeago: 8, vista ventral; 9, vista lateral; 10, vista dorsal (g, gancho; r.sa, rama subapical).

Terminalia. Cerci parcialmente unidos al epandrio. Epandrio microtricoso en la parte dorsal y desnudo en la parte dorsal media, con 11 cerdas en la parte baja en ambos lados (lado derecho roto). Decasterno triangular. Surestilo con 13 dientes primarios en ambos lados, ocho cerdas marginales en el lado izquierdo y siete en el lado derecho (Fig. 6). Hipandrio de forma triangular poco quitinizado y membranoso. Gonopodio poco quitinizado, alargado, con una cerda en la parte interna (Fig. 7). Edeago poco quitinizado. Parte distal del edeago triangular, con la punta ligeramente hendida, en vista ventral con dos proyecciones laterales pequeñas como ganchos (g), y un par de espuelas largas y delgadas (r.sa) (Figs 8-10). En vista dorsal el borde del edeago quitinizado y la parte central menos quitinizado. Rama ventral quitinizada poco desarrollada. Paráfisis larga con una cerda en el externo distal. Pie del edeago poco quitinizado. Índice del edeago 3.40.

Variación en paratipos (individuos en alcohol).
Morfología externa: longitud total (cuerpo + alas) 4.16$4.26 \mathrm{~mm}$. Longitud frontal $0.38-0.41 \mathrm{~mm}$; índice frontal 1.21-1.24; radio de disminución frontal 1.62-1.66; índice vt $0.93-0.95$; distancia or 1/or3 0.11-0.12; distancia or2/or1 0.05-0.07; índice genal 3.70-3.73; índice vibrisa 0.62-0.65; índice ocular 1.01-1.02. Algunos individuos pueden ser más oscuros en la parte anterior del tórax. Índice h 1.001.02; índice dc 0.74-0.75; índice scut 1.40-1.42; índice sterno $0.76-0.79$. Algunos paratipos con el primer tergito no pigmentado. Epandrio, algunos especímenes presentan pelos y cerdas, de 10-14, en la parte media y baja del epandrio. Surestilo con 12-13 dientes primarios, siete-10 cerdas marginales. Índice del edeago 3.39-3.40.

Hembra: desconocida.

Etimología. "Nigua" es el nombre de uno de los primeros pueblos nativos del grupo étnico Colorados que habitaron en "Santo Domingo de los Colorados" hoy conocido como la Provincia de "Santo Domingo de los 
Tsáchilas", lugar de captura de esta especie.

Relación con otras especies. D. nigua sp. nov. ha sido agrupada en el subgrupo $D$. fasciola, debido a las características que comparte con las especies del subgrupo, especialmente la presencia de un par de espuelas subapicales en la región ventral del edeago, además ha sido capturada en bosques nublados como las especies de este subgrupo (Tosi et al., 1990; Costa \& Sene, 2002; Silva-Bernardi et al., 2006).

\section{Drosophila yambe sp. nov.}

(Figs 11-15)

Material tipo. Holotipo $\widehat{\partial}$ (disectado, genitalia en microtubo, montado en seco), ECUADOR, Pichincha: (Intillacta, 0003'01.6”'N 07843'23.7' W, 1890 msnm), II.2011, M. B. Cabezas col., V. Rafael \& M. B. Cabezas

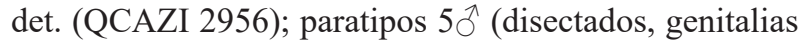
en microtubo, montados en seco) los mismos datos del holotipo, (QCAZI 2957-2961). Paratipos 2ð ECUADOR, Santo Domingo de los Tsáchilas: (Guajalito, $00^{\circ} 13^{\prime} 48.8^{\prime \prime} \mathrm{S}$, 078 49'16.5”W, 1890 msnm) VII.2011, M. B. Cabezas col., V. Rafael \& M. B. Cabezas det. (QCAZI 2962-2953).

Diagnosis. Color del cuerpo amarrillento. Tórax con cerdas y pelos emergiendo de manchas oscuras. Abdomen amarillo, con línea media dorsal del segundo al sexto tergito. En todos los tergitos con una pigmentación en la parte posterior que se adelgaza hacia los lados, luego la pigmentación alcanza el borde superior de los tergitos seguido por un espacio amarillo y nuevamente el tergito se pigmenta a manera de triángulo. Edeago en forma de $\mathrm{C}$, del centro del edeago emerge una lámina membranosa (l.m) apenas perceptible; en vista dorsal ápice del edeago ligeramente hendido y con una rama subapical larga y delgada (r.sa).

Descripción del macho, holotipo (individuo en alcohol). Morfología externa: longitud total (cuerpo + alas) $4.20 \mathrm{~mm}$, longitud del cuerpo $2.70 \mathrm{~mm}$. Color del cuerpo amarillento.

Cabeza. Marrón con áreas amarillas. Segundo y tercer segmento de las antenas marrón amarillento. Arista plumosa, con cuatro ramas dorsales y dos ventrales, más la terminal bifurcada. Placa orbital marrón, longitud frontal $0.50 \mathrm{~mm}$; índice frontal 1.61; radio de disminución frontal 1.74; la cerda orbital media más cerca a la cerda orbital anterior y del borde de la placa orbital, nacen de puntos oscuros, índice vt 0.81 ; distancia or $1 /$ or 30.27 ; distancia or $2 /$ or1 distancia 0.11 . Triángulo ocelar marrón oscuro, ocelos amarillos; triángulo frontal, frontal vitta, gena y postgena marrón, índice genal 3.75 ; índice vibrisa 0.44 . Una cerda oral prominente. Carina ligeramente surcada color marrón amarillento. Proboscis y palpo marrón amarillentas. Ojos de color rojo vinoso, índice ocular 1.15.

Tórax. Amarillo, con cerdas y pelos emergiendo de manchas oscuros, con seis hileras de pelos acrosticales entre las cerdas dorsocentrales anteriores, índice h 0.83 ; índice de 0.67, a lo largo de todo el tórax en dirección de las dorsocentrales hay una banda oscura, parte central del tórax ligeramente más oscura, el lóbulo prescutal amarillo rodeado con una zona oscura. Escutelo color marrón amarillento; cerdas escutelares anteriores convergentes emergiendo de puntos oscuros, índice scut 1.37; índice sterno 0.78. Patas coxa y fémur marrón oscuro, tibia presenta dos anillos uno en la parte inicial y otro en la parte final. Alas amarillas, vena transversal posterior poco sombreada, lóbulo costal oscuro. Longitud del ala 3.05 mm. Índices alares: alar 1.83; C 2.29; ac 2.46; hb 0.51; 4c $1.14 ; 4 \mathrm{v} 1.94 ; 5 \mathrm{x} 1.25 ; \mathrm{M} 0.53$ y prox. x 0.73 .

Abdomen. Color amarillo, primer tergito no pigmentado, con línea media dorsal del segundo al sexto tergito. Todos los tergitos con pigmentación en la parte posterior que se adelgaza hacia los lados. Luego la pigmentación alcanza el borde anterior de los tergitos seguido por un espacio amarillo y nuevamente el tergito se pigmenta a manera de triángulo.

Terminalia. Cerci parcialmente unidos al epandrio (roto). Epandrio microtricoso y con seis cerdas en la parte dorsal y 15 cerdas en la parte media y baja, poco microtricoso. Decasterno ancho. Surestilo con 11 dientes primarios en ambos lados, nueve cerdas marginales en ambos lados, el lado derecho presenta una cerda marginal en la parte media del surestilo (Fig. 11). Hipandrio en forma de copa muy quitinizado, parte posterior membranoso. Gonopodios quitinizados con forma $\mathrm{C}$, con una cerda en la parte baja del gonopodio (Fig. 12). Edeago quitinizado. En vista lateral, edeago en forma de C, del centro del edeago emerge una lámina membranosa (1.m) apenas perceptible, el contorno distal del edeago así como las ramas subapicales (r.sa) presentan granulaciones. En vista dorsal, parte distal del edeago de forma triangular, ápice ligeramente hendida, los extremos laterales terminan en punta y con un par de espuelas alargadas delgadas muy quitinizadas que se entrecruzan (r.sa). La abertura dorsal nace en la base del edeago y alcanza 3/4 partes del edeago (Figs 13-15). Paráfisis alargada, con el extremo distal en forma de dedo, microtricosa y con una cerda. Pie del edeago quitinizado corto. Índice del edeago 2.05.

Variación en paratipos (individuos en alcohol). Morfología externa: longitud total (cuerpo + alas) 3.93$4.21 \mathrm{~mm}$. Longitud frontal $0.49-0.53 \mathrm{~mm}$; índice frontal 1.60-1.64; radio de disminución frontal 1.72-1.76; índice vt $0.79-0.83$; distancia or1/or3 $0.27-0.29$; distancia or $2 /$ or1 $0.11-0.14$; índice genal $3.68-3.75$; índice vibrisa 0.44 0.46; índice ocular1.13-1.20. Índice h 0.81-0.83; índice dc 0.64-0.68; índice scut 1.37-1.40; índice sterno 0.770.79 . Epandrio parte media y baja con cuatro-siete pelos (algunos individuos no son microtricosos en la parte baja). Surestilo con 10-13 dientes primarios, ocho-nueve cerdas marginales. Edeagos pálidos en individuos jóvenes. Índice del edeago 2.03-2.07.

Hembra: desconocida.

Etimología. "Yambe" es el nombre de uno de los primeros pueblos nativos del grupo étnico Colorados que 


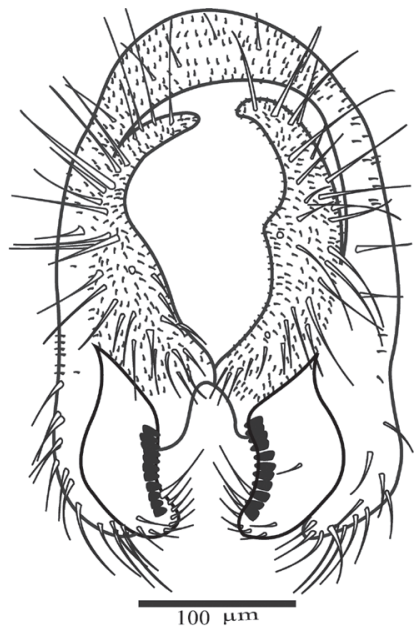

11

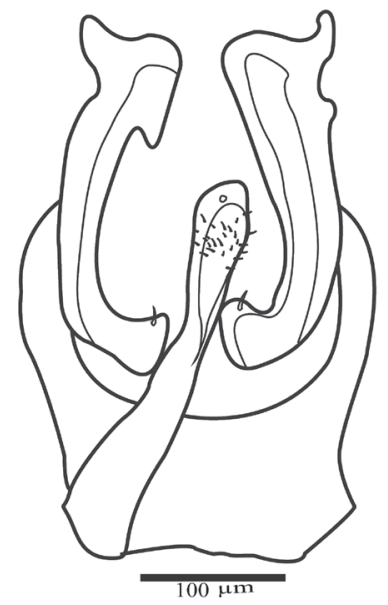

12
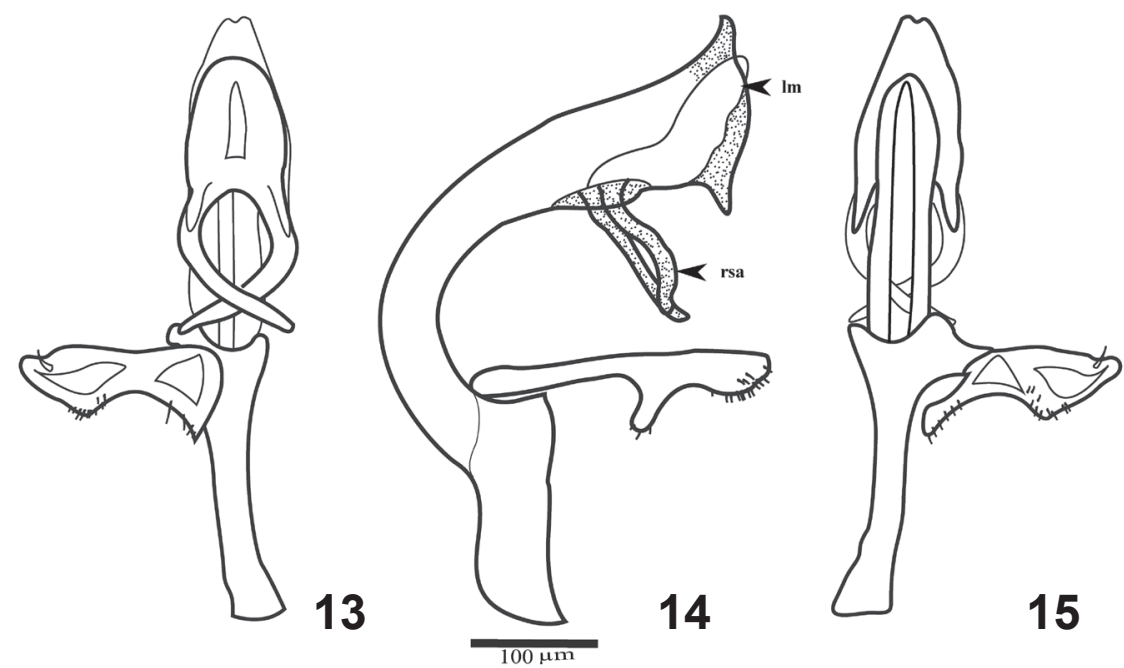

Figs 11-15. Drosophila yambe sp. nov., holotipo ô: 11, arco genital; 12, hipandrio; edeago: 13, vista ventral; 14, vista lateral; 15, vista dorsal (1.m, lámina membranosa; r.sa, rama subapical).

habitaron en "Santo Domingo de los Colorados" hoy conocido como la Provincia de "Santo Domingo de los Tsáchilas", lugar de captura de esta especie.

Relación con otras especies. D. yambe sp. nov. ha sido agrupada en el subgrupo $D$. fasciola, debido a la presencia de un par de espuelas en la región ventral del edeago, característica propia del subgrupo $D$. fasciola. Esta especie también ha sido capturada en bosques nublados como las especies del subgrupo D. fasciola (Tosi et al., 1990; Costa \& SENE, 2002; Silva-Bernardi et al., 2006).

\section{Drosophila carvalhoi sp. nov.}

(Figs 16-22)

Material tipo: Holotipo $\hat{\delta}$ (disectado, genitalia en microtubo, montado en seco), ECUADOR, Santo Domingo de los Tsáchilas: (Guajalito, $00^{\circ} 13^{\prime} 48^{\prime}$ 'S 7849’16”W,
1890), VIII.2011, L. M. Llangarí col., V. Rafael \& L. M. Llangarí det. (QCAZI 2975); paratipo 15^ (disectado, genitalia en microtubo, montado en seco) ECUADOR, Santo Domingo de los Tsáchilas: los mismos datos del holotipo, VII.2011 (QCAZI 2976-2990). Alotipo ㅇ (disectado, genitalia en microtubo, montado en seco), ECUADOR, Santo Domingo de los Tsáchilas: los mismos datos del holotipo (QCAZI 2991); paratipo 3 (disectado, genitalia en microtubo, montado en seco) ECUADOR, Santo Domingo de los Tsáchilas: los mismos datos del holotipo, VIII.2011 (QCAZI 2992), VII.2011 (QCAZI 2993-2994).

Diagnosis. Color del cuerpo marrón. Tórax con cerdas y pelos emergiendo de manchas oscuras. Abdomen amarillo con línea media dorsal, del segundo tergito al sexto tergito con una franja marrón en el borde posterior que alcanza la mitad del tergito y en la parte lateral la 

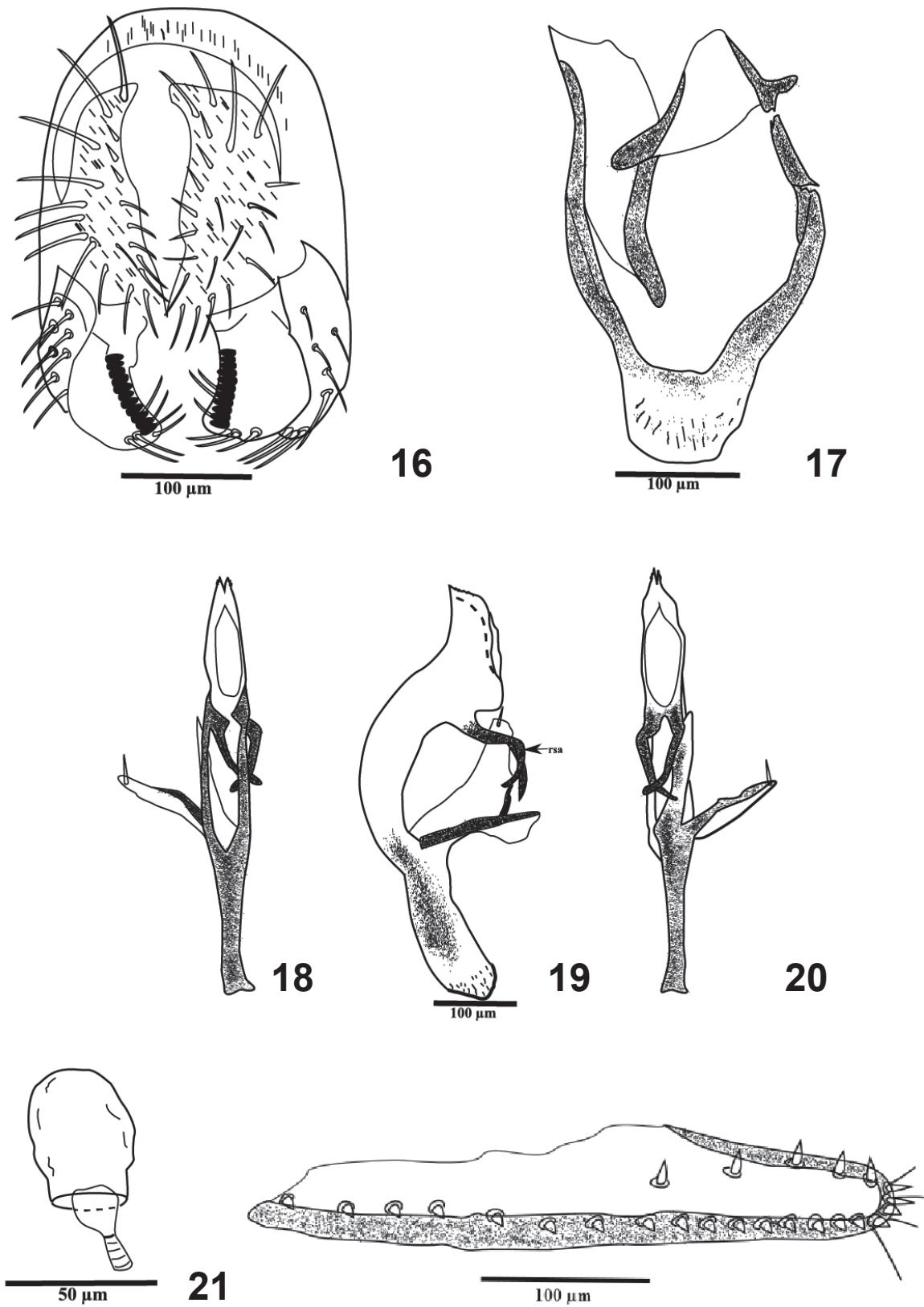

22

Figs 16-22. Drosophila carvalhoi sp. nov. Holotipo $\lesssim$ : 16, epandrio; 17, hipandrio; edeago: 18, vista dorsal; 19, vista lateral; 20, vista frontal. Alotipo ㅇ: 21 , espermateca; 22 , oviscapto (r.sa, rama subapical).

franja alcanza el borde anterior, donde nace una línea diagonal clara que alcanza el borde posterior. Parte distal del edeago bífido y ligeramente aserrado, con un par de espuelas subapicales quitinosas largas y delgadas.

Descripción del macho, holotipo (individuo en alcohol). Morfología externa: longitud total (cuerpo + alas) $3.30 \mathrm{~mm}$, longitud del cuerpo $2.70 \mathrm{~mm}$. Color del cuerpo marrón.

Cabeza. Segundo segmento antenal ligeramente oscuro en la parte dorsal y más claro en la parte ventral; tercer segmento antenal marrón claro polinoso, arista plumosa con cuatro ramas dorsales y dos ventrales, más la terminal bifurcada. Placa orbital marrón amarillento, longitud frontal $0.35 \mathrm{~mm}$; índice frontal 1.20 ; radio de disminución frontal 1.65; la cerda orbital media más cerca de la cerda orbital anterior y ligeramente hacia el borde exterior de la placa orbital, índice vt 0.98 ; distancia or $1 /$ or3 0.08 ; distancia or $2 /$ or 10.03 . Triángulo frontal marrón claro; triángulo ocelar marrón oscuro, ocelos amarillos. Frontal vitta marrón oscuro. Gena y postgena marrón, índice genal 3.00; índice vibrisa 0.42 . Carina marrón claro, ligeramente prominente y surcada. Proboscis marrón amarillento. Ojos de color rojo vinoso, índice ocular 1.02 .

Tórax. Marrón amarillento con seis hileras de pelos 
acrosticales entre las cerdas dorsocentrales anteriores, índice h 0.76 ; índice dc 0.81 . Las cerdas y pelos emergen de puntos marrón oscuro. Con una franja más clara en el centro y a lo largo de las dorsocentrales con otra franja más oscura. Escutelo marrón amarillento. Cerdas escutelares anteriores convergentes emergiendo de puntos marrón oscuro, índice scut 0.82 ; índice sterno 0.28 . Pleura marrón. Patas con la coxa y fémur marrón oscuro, tibia amarilla con dos anillos de color marrón uno en la parte proximal y otro en la parte distal. Alas amarillas, lóbulo costal oscuro. Longitud del ala $2.38 \mathrm{~mm}$. Índices alares: alar 2.10; C 2.40 ; ac 2.50; hb 0.60; 4c 0.90; 4v 1.60; 5x 1.30; M 0.50 y prox. x 0.60 .

Abdomen. Amarillo con línea media dorsal. Primer tergito marrón; del segundo al sexto tergito con una franja marrón en el borde posterior que alcanza la mitad del tergito y en la parte lateral la franja alcanza el borde anterior, donde nace una línea diagonal clara que alcanza el borde posterior (en otros individuos alcanza la mitad del tergito).

Terminalia. Cerci parcialmente unidas al epandrio. Epandrio con finos pelos en la parte superior; con ocho cerdas en la parte baja izquierda y derecha. Surestilo rectangular, ambos lados con 11 dientes primarios y seis cerdas marginales (Fig. 16). Hipandrio (roto) quitinizado en forma de "V", gonopodio poco quitinizado (Fig. 17). Edeago poco quitinizado, parte distal bífido y ligeramente aserrado, con un par de espuelas subapicales quitinizadas, largas y delgadas (r.sa) (Figs 18-20). Rama ventral poco desarrollada. Paráfisis larga con una cerda en el extremo distal. Índice del edeago 1.50

Variación en paratipos $\widehat{\partial}$ (individuos en alcohol). Morfología externa: longitud total (cuerpo + alas) 3.30$4.10 \mathrm{~mm}$. Longitud frontal $0.35-0.41 \mathrm{~mm}$; índice frontal 1.20-1.24; radio de disminución frontal 1.63-1.67; índice vt $0.97-1.00$ ); distancia or1/or3 0.07-0.08; distancia or $2 /$ or1 0.03; índice genal 2.98-3.06); índice vibrisa 0.410.43 ; índice ocular 1.01-1.22. Índice h 0.75-0.78; índice dc $0.80-0.83$; índice scut $0.80-0.83$; índice sterno 0.26 0.30 . Surestilo rectangular, izquierdo y derecho con 10-12 dientes primarios y seis-siete cerdas marginales. Índice del edeago 1.48-1.51.

Descripción de la hembra, alotipo y paratipos (provenientes de isolínea, individuos en alcohol). Morfología externa alotipo: longitud total (cuerpo + alas) $3.70 \mathrm{~mm}$, longitud del cuerpo $3.20 \mathrm{~mm}$. Color del cuerpo marrón.

Cabeza. Similar al macho excepto en la arista que presenta tres ramas dorsales y dos ventrales, más la terminal bifurcada.

Tórax. Similar al macho. Longitud del ala: 2.30 mm. Índices alares: alar 2.00; C 2.10; ac 2.40; hb 0.40; 4c $1.10 ; 4 \mathrm{v} 1.70 ; 5 \mathrm{x} 1.20 ; \mathrm{M} 0.50$ y prox. x 0.60 .

Abdomen. Similar al macho.

Terminalia. Oviscapto alargado, estrecho, triangular y apicalmente redondeado con cinco ovisensillas discales con la punta redondeada y 20 ovisensillas marginales; tres ovisensillas internas como tricomas y una subterminal, larga y ligeramente curvada (Fig. 22). Espermateca redondeada, membranosa y rugosa (Fig. 21). Índice de la espermateca 1.6.

Variación en paratipos 9 . Morfología externa: longitud total (cuerpo + alas) 4.01-4.23 mm. Oviscapto con cinco-siete ovisensillas discales y 19-20 ovisensillas marginales. Índice de la espermateca 1.4-1.6.

Ecología. Individuos capturados en las inflorescencias de Anthurium sp.

Relación con otras especies. Drosophila carvalhoi sp. nov. estaría relacionada con Drosophila nigua sp. nov., por presentar bandas más oscuras a lo largo del tórax y por la forma del edeago

Etimología. En honor al Dr. Bernardo Carvalho de la Universidad de Rio de Janeiro, Brasil.

\section{DISCUSIÓN}

El grupo D. repleta es endémico de las regiones semiáridas del continente Americano (PEREIRA et al., 1983); consta de 102 especies descritas y ubicadas en varios subgrupos (GotTsCHALK et al., 2006). Uno de los subgrupos es $D$. fasciola que comprende 21 especies, las cuales viven principalmente en bosques húmedos, pero se conoce poco sobre el sustrato al que están asociadas (Pereira et al., 1983; Rafael \& Arcos, 1989).

En el presente trabajo se describen cuatro miembros nuevos del subgrupo $D$. fasciola. Los cuatro nuevos miembros fueron capturados en bosques nublados como la mayoría de las especies del subgrupo $D$. fasciola (TosI et al., 1990; Costa \& SENE, 2002; SiLVA-Bernardi et al., 2006). La identificación de estas especies se basó en el patrón de pigmentación del tórax y la morfología de la genitalia del macho.

Las especies $D$. inti sp. nov. y D. linearepleta se supone que estarían emparentadas debido a las semejanzas morfológicas de la genitalia. Sin embargo, existen diferencias, $D$. inti sp. nov. tiene el edeago ligeramente más ancho y con estrías en la parte dorsal, mientras que $D$. linearepleta tiene el edeago menos ancho y con pequeñas microproyecciones en la parte ventral.

Drosophila linearepleta, encontrada en Guatemala y México, estaría relacionada con $D$. hermioneae Vilela, 1983, registrada en México, y D. fulvalineata Patterson \& Wheeler, 1942 (VILELA, 1983), capturada en Arizona y en zonas del desierto mexicano. Por lo tanto, $D$. inti sp. nov. al compartir la forma básica del edeago, además de algunas características de la genitalia externa, podría ser la cuarta especie de un complejo de especies del subgrupo D. fasciola.

Drosophila nigua sp. nov. y D. yambe sp. nov. han sido agrupadas también en el subgrupo $D$. fasciola, por las características que comparten con las especies del subgrupo, especialmente la presencia de un par de espuelas subapicales en la región ventral del edeago.

Drosophila carvalhoi sp. nov., encontrada en el bosque nublado de Guajalito, también sería un miembro 
nuevo del subgrupo $D$. fasciola. Esta especie tiene semejanza con $D$. nigua sp. nov. y $D$. yambe sp. nov. en la pigmentación del abdomen, la forma general del edeago, punta bífida, un par de espuelas subapicales y paráfisis alargada.

Drosophila inti sp. nov., D. nigua sp. nov. y $D$. yambe sp. nov. fueron capturadas en trampas con banano fermentado, por lo que se puede presumir que los sitios de alimentación y cría están relacionados con frutos en descomposición como ya se ha registrado en algunas especies del grupo $D$. repleta en trabajos anteriores en bosques andinos (RAFAEL et al., 2000).

Algunas especies del subgrupo D. fasciola se alimentan de flores marchitas y frutos caídos como es el caso de $D$. fasciola, que se alimenta y se reproduce en las flores caídas de Aphelandra micans Moritz ex Vatke, 1876, Erythrina berteroana Urban, 1908, Heliconia latispatha Bentham, 1846, Aroid sp. y frutos de Coffea arabica Linnaeus, 1753 (PIPKIN et al., 1966). Drosophila carvalhoi sp. nov. fue capturada en inflorescencias de Anthurium sp. Aunque no se dispone de más datos sobre los sitios de cría y alimentación de $D$. carvalhoi sp. nov., cabe mencionar que simultáneamente, durante cuatro colectas, se colocaron trampas de banano fermentado en el bosque y $D$. carvalhoi sp. nov. jamás fue capturada en las trampas, por lo tanto, es probable que esta especie este restringida a flores del género Anthurium sp.

Agradecimientos. A la Pontificia Universidad Católica del Ecuador por financiar los proyectos: $\mathrm{N}^{\circ}$. H13171, $\mathrm{N}^{\circ}$. H19121 y $\mathrm{N}^{\circ}$. J13054. A Inti Arcos y su esposa Nina Duarte por brindarnos las facilidades para realizar las colectas en la Reserva Intillacta. A Elena Grijalva por permitirnos realizar las colectas en la Estación Científica Río Guajalito. Al ministerio del ambiente por otorgar los permisos de colección $\mathrm{N}^{\circ}$ 001-10 IC-FAU-DNB/MA y No 001-11 IC-FAU-DNB/MA. A Diego Céspedes y Luna Figuero por su ayuda en la fase de campo.

\section{REFERENCIAS BIBLIOGRÁFICAS}

Acurio, A. E. \& Rafael, V. L. 2009. Inventario taxonómico de Drosophilidae (Diptera) en el parque Nacional Yasuní, Amazonia Ecuatoriana. Acta Amazonica 39(3):713-718.

Bächli, G.; Vilela, C. R.; Andersson, S. \& Saura, A. 2004. The Drosophilidae (Diptera) of Fennoscandia and Denmark. Vol. 39. Leiden, New York, Ed. Brill. 362p.
Cavasini, R.; Pincela, M. R. \& Tunes, B. M. L. 2008. Caracterização da assembléia de Drosophila (Diptera, Drosophilidae) em dois fragmentos florestais na região sul do Brasil. Revista Electrônica Lato Sensu 3(1):1980-6116.

Costa, C. T. A. \& Sene, F. M. 2002. Characterization of courtship sounds of species of the subgroup fasciola (Diptera, Drosophilidae, Drosophila repleta group): interspecific and interpopulational analyses. Brazilian Journal of Biology 62(4A):573-583.

Dangles, O.; Nowicki, F. \& Mena, B. 2009. Biota Maxima: Ecuador Biodiverso. Quito, Imprenta Mariscal, Pontificia Universidad Católica del Ecuador. 250p.

Gottschalk, M. S.; Döge, J. S.; Oliveira, S. C. F.; De Toni, D. C.; Valente, V. L. S. \& Hofmann, P. R. P. 2006. On the geographical distribution of the Drosophila subgenus in southern Brazil (Drosophiliade, Diptera). The $D$. repleta species group. Tropical Zoology 19:129-139.

Pereira, M. A. Q. R.; Vilela, C. \& Sene, F. 1983. Notes on breeding and feeding sites of some species of the repleta group of the Genus Drosophila (Diptera, Drosophilidae). Ciência e Cultura 35:13131319.

PiPkIN, S.; Rodríguez, R. \& León, J. 1966. Plant host specificity among Flower-Feeding Neotropical Drosophila (Diptera: Drosophilidae). American Naturalist 100:135-156.

Rafael, V. \& Arcos, G. 1989. Subgrupo inca, un nuevo subgrupo del grupo repleta, con descripción de Drosophila huancavilcae n. sp. (Diptera, Drosophilidae). Evolución Biológica 3(3):233-243.

Rafael, V.; Arcos, G. \& Arcos Terán, L. 2000. Ecología y distribución del género Drosophila en Guayllabamba y el Quinche, provincia de Pichincha - Ecuador. Revista de la Pontificia Universidad Católica 65:130-155.

Silva-Bernardi, E. C. C.; Morales, A. C.; Sene, F. M. \& Manfrin, M. H. 2006. Phylogenetic relationships of the Drosophila fasciola species subgroup (Diptera, Drosophilidae) inferred from partial sequences of the mitrochondrial cytochrome oxidase subunit I (COI) gene. Genetics and Molecular Biology 29(3):566-571.

Suyo, M. P. \& Pilares, G. L. 1988. Drosophila novemaristata: descripción, taxonomía y nuevas localidades peruanas. Revista Peruana de Entomología 30:61-64.

Suyo, M. P.; Pilares, G. L. \& VÁsquez, E. J. 1988. Genitalia externa de Drosophila huayla sp. nov., Cluster Mulleri. Revista Peruana de Entomología 30:58-60.

Throckmorton, L. 1982. The virilis species group. In: AsHBURner, M.; Carson, H. L. \& Thompson, J. N. eds. The genetics and biology of Drosophila. London, Academic Press, p. 227-297.

Tosi, D.; Martins, M.; Vilela, C. R. \& Pereira, M. A. Q. R. 1990. One a new cave-dwelling species of bat-guano-breeding Drosophila closely related to D. repleta Wollaston (Diptera, Drosophilidae). Revista Brasileira de Genetica 13(1):19-31.

Vilela, C. R. 1983. A revision of the Drosophila repleta species group (Diptera, Drosophilidae). Revista Brasileira de Entomologia 27(1):1-114. 